\title{
A large catalog of young active RAVE stars in the Solar neighborhood
}

\author{
Maruša Žerjal ${ }^{1}$, Tomaž Zwitter ${ }^{1}$, Gal Matijevič̃ ${ }^{2}$ and RAVE \\ Collaboration $^{3}$ \\ ${ }^{1}$ Faculty of Mathematics and Physics, University of Ljubljana, \\ Jadranska 19, 1000 Ljubljana, Slovenia \\ email: marusa.zerjal@fmf .uni-lj.si \\ ${ }^{2}$ Leibniz-Institut für Astrophysik Potsdam (AIP), \\ An der Sternwarte 16, D-14482, Potsdam, Germany \\ ${ }^{3}$ https://www.rave-survey.org/
}

\begin{abstract}
The catalog of 38,000 chromospherically active RAVE dwarfs represents one of the largest samples of young active solar-like and later-type single field stars in the Solar neighbourhood. It was established from the unbiased magnitude limited RAVE Survey using an unsupervised stellar classification algorithm based merely on stellar fluxes (Ca II infrared triplet). Using a newly-calibrated age-activity relation, $\sim 15,000$ active stars are estimated to be younger than 1 Gyr. Almost 2000 stars are presumably younger than $100 \mathrm{Myr}$ and possibly still in the pre-main sequence phase, the latter being supported by their significant offset from the main sequence in the $N_{U V}-V$ versus $J-K$ space. 16,000 stars from the sample have positional and velocity vectors available (using TGAS parallaxes and proper motions and radial velocities from RAVE).
\end{abstract}

Keywords. stars: activity, chromospheres, emission-line, Be, pre-main-sequence, catalogs

\section{Automated search for active field stars in large spectroscopic surveys}

The latest RAVE data release (DR5, Kunder et al. 2016) includes 521,000 spectra of 458,000 southern sky stars with an unbiased magnitude limited selection function $(9<I<12)$. Due to the large number of spectra, automated, possibly parameter free classification techniques are necessary to discover peculiar objects, e.g., active stars. Their additional flux in the strongest spectral lines (e.g., Ca II IRT in RAVE) makes chromospherically active stars easily recognized by spectral classification techniques, such as locally linear embedding (LLE). Because LLE is a general dimensionality reduction procedure that conserves relations between the neighboring points of the high-dimensional manifold, a selected spectrum in the projected (2D) space is surrounded by its neighbors from the high-dimensional space (for more details on classification of the RAVE spectra see Matijevič et al. 2012). In view of a single observation with a moderate signal-tonoise ratio $(\mathrm{S} / \mathrm{N}>20)$ and a mid-range resolution being sufficient this approach enables the discovery of a vast number of young, chromospherically active field stars in large spectroscopic surveys.

In RAVE, 38,000 dwarfs were recognized as active candidates (Žerjal et al. 2013) with activity levels continuously increasing from marginally active stars to individual cases with emission peaks exceeding the continuum level. Many of the most active stars from the sample show strong X-ray (ROSAT) and ultraviolet excess emission in addition to their position off the main sequence in the $J-K$ (2MASS) versus $N_{U V}-V$ diagram 


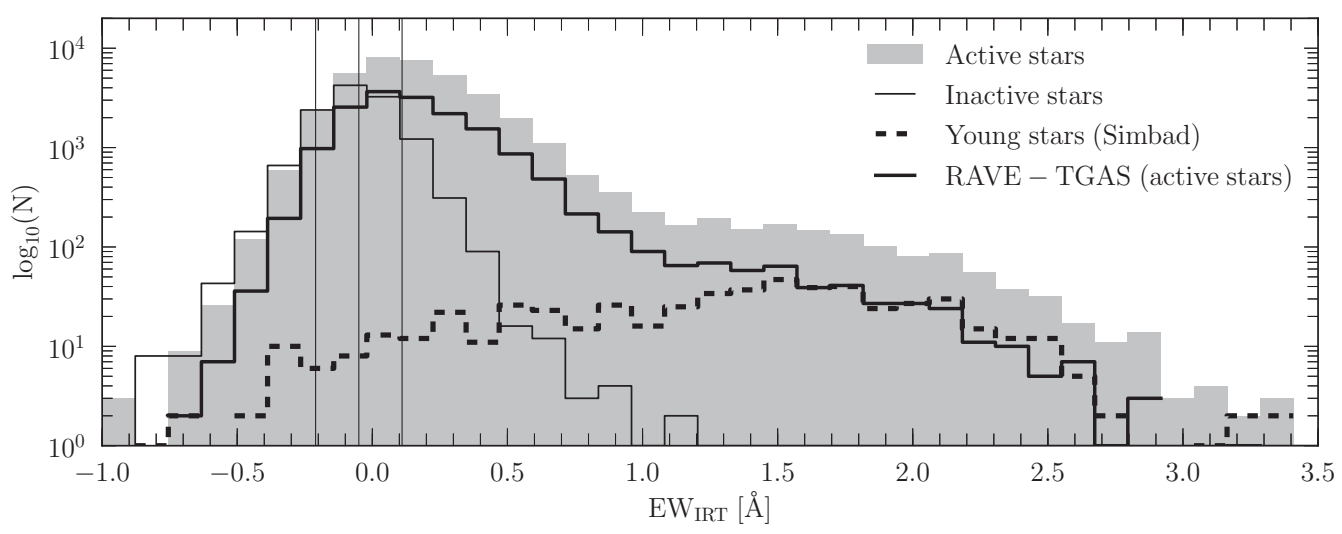

Figure 1. The distribution of activity levels is bimodal: there is 22,000 stars (58\%) above $1 \sigma$ and 7000 stars $(18 \%)$ above $3 \sigma$ activity detection level. Stars marked as young in the Simbad database (e.g., T Tau, Young, Pre-main sequence objects) mostly coincide with the more active peak of the distribution. Vertical lines in the plot mark $\pm 1 \sigma$ deviation. Image adapted from Zerjal et al. 2016.

(using $N_{U V}$ from GALEX and $V$ from APASS) where they overlap with the reference pre-main sequence RAVE stars (according to the Simbad database).

\section{Parameter-free data-driven characterization of activity levels in the Ca II IRT}

In order to quantitatively characterize emission levels a large database of 12,000 spectra of inactive RAVE dwarfs was used as a template library. After the cores of the calcium lines were removed $( \pm 2.5 \AA$ from the center $)$ from both active and inactive spectra, each normalized active spectrum was compared to the inactive database to find its nearest neighbors. Thanks to the large database of inactive stars covering the entire parameter space (effective temperature, surface gravity, metallicity etc.) it was possible to find inactive counterparts with the same stellar parameters for each active spectrum. The procedure was parameter-free: no atmospheric parameters were used to avoid biases originating from the pipeline designed for inactive dwarfs and giants. Another advantage of the measured inactive template database over synthetic spectra is the absence of the non-LTE problems in the cores of the calcium lines where emission is present.

The sum of the equivalent widths of the disentangled emission flux $\mathrm{EW}_{\mathrm{IRT}}=\mathrm{EW}_{8498}+$ $\mathrm{EW}_{8542}+\mathrm{EW}_{8662}$ is used as a proxy for activity levels (Žerjal et al. 2013, Figure 1). No photospheric correction is needed and the metallicity term is reduced. The typical uncertainty of the $\mathrm{EW}_{\mathrm{IRT}}$ estimation is $0.16 \AA$.

\section{Age-activity relation}

It is well known that stellar activity diminishes with age (e.g., Mamajek \& Hillenbrand 2008). Age estimates from the literature for 137 active RAVE stars (mostly cluster, moving group and association members) enabled the age-EW $\mathrm{EW}_{\mathrm{IRT}}$ calibration in the range from $\sim 1$ Gyr down to a few 10 Myr (Žerjal et al. 2016, Figure 2). Although the scatter is large due to time variability, the saturation of activity in the youngest stars and age uncertainties, the anti-correlation between the two is clear. The data are divided into three main age-activity regimes: $\geqslant 1$ Gyr $\left(\mathrm{EW}_{\text {IRT }}<0.25 \AA\right)$, between 0.1 and $1 \operatorname{Gyr}\left(0.25 \AA<\mathrm{EW}_{\text {IRT }}<0.75 \AA\right)$ and younger than $100 \mathrm{Myr}\left(\mathrm{EW}_{\text {IRT }} \geqslant 0.75 \AA\right)$. 


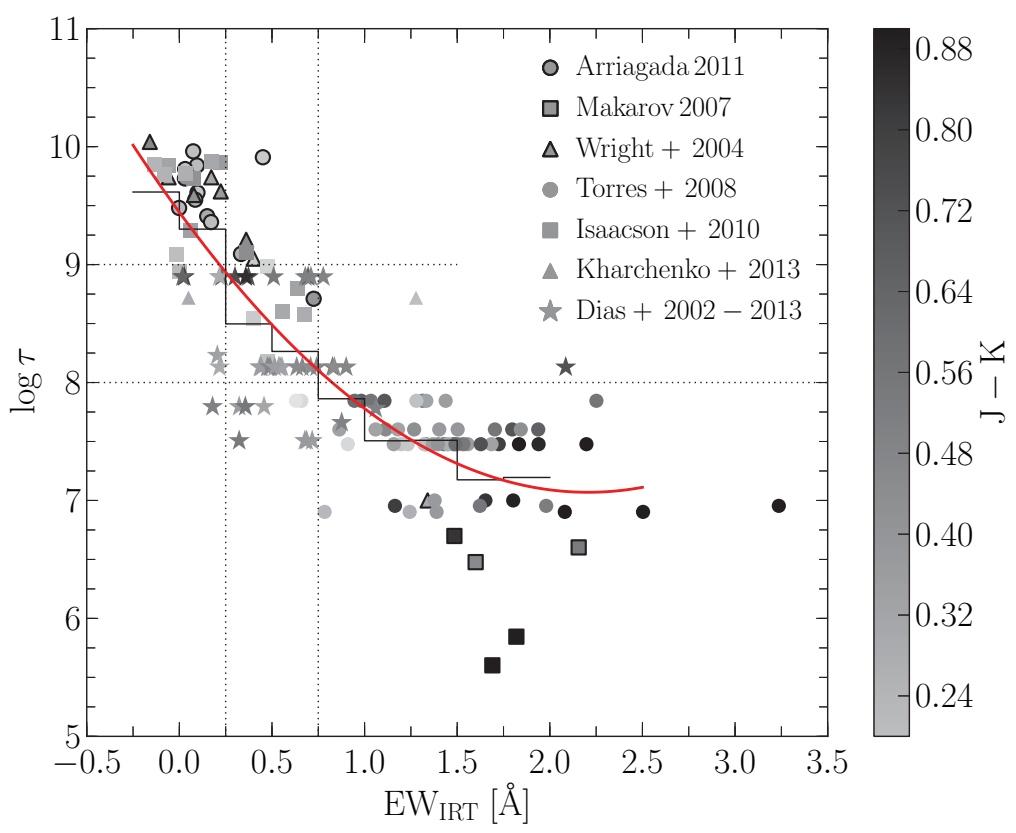

Figure 2. Age-EW $\mathrm{EW}_{\mathrm{IRT}}$ activity calibration using 137 RAVE dwarfs with known ages. Most of the stars are cluster, moving group and association members. Although the scatter is large the correlation is clear. Thin black line shows an averaged age for a given activity bin while thick solid (red) line is a parabolic fit to the data. Dotted lines mark the three main activity-age classes. Figure adapted from Žerjal et al. 2016.

$\sim 15,000$ RAVE field dwarf stars are shown to be younger than 1 Gyr and $\sim 2000$ younger than 100 Myr.

A combination of an efficient automated stellar classification algorithm and the ageactivity relation offers an opportunity to build a young active candidate database and to perform further follow-up studies of dwarfs that possibly harbour exoplanets. The active database will be vastly enhanced with emission catalogs from the ongoing and future surveys, e.g. Galah (De Silva et al. 2015), FunnelWeb (Lawson et al. 2016) and Gaia (covers Ca II IRT as well).

\section{References}

De Silva, G. M., Freeman, K. C., Bland-Hawthorn, J., et al. 2015, MNRAS, 449, 2604

Kunder, A., Kordopatis, G., Steinmetz, M., et al. 2016, arXiv:1609.03210

Lawson, W., Murphy, S., Tinney, C. G., Ireland, M., \& Bessell, M. S. 2016, American Astronomical Society Meeting Abstracts, 228, 217.08

Mamajek, E. E. \& Hillenbrand, L. A. 2008, ApJ, 687, 1264-1293

Matijevič, G., Zwitter, T., Bienaymé, O., et al. 2012, ApJS, 200, 14

Žerjal, M., Zwitter, T., Matijevič, G., et al. 2013, ApJ, 776, 127

Žerjal, M., Zwitter, T., Matijevič, G., et al. 2016, ApJ, accepted. 